\title{
Scheduling of processes and results of the reorganization of construction objects
}

\author{
Ruben Kazaryan ${ }^{1, *}$ \\ ${ }^{1}$ Moscow State University of Civil Engineering, 26 Yaroslavskoe shosse, Moscow, 129337, Russia
}

\begin{abstract}
The paper discusses the feasibility and necessity of modeling technogenic impacts as an important element of managing the construction reorganization of objects in order to ensure the competitiveness of the proposed organizational and technological solutions, for the purpose of the scientific research and virtual modeling of a construction object and construction industry that erects or reorganizes. The result of a study is the obtaining and introduction of new methods of organizing labor activity and its elements into the practice of construction industry, specifically, the analysis of existing domestic and foreign developments of virtual models and methods of organizational and technological design for erection or constructional reorganization of buildings and structures; study of o control system characteristics; simulation of the objective function; modeling of the objective function (criterion) of management at the pre-project stage of erection and constructional reorganization of buildings and structures; development of organizational structures and technologies of construction; formation of a methodology for the development of elements of technical, technological and organizational support for the management. Using such diagnostic models, time series of predicted retrospective values of parameters of technogenic impacts are built. Visual displays of such time series (infographic models) are known as "trends".
\end{abstract}

\section{Introduction}

Scientific and technical progress and increasing in volume and complexity requirements for the functioning of the domestic construction industry determine the current trends of construction science [1-5]:

Development of competitive new technologies and improvement of existing ones, development of organizational forms and structures, methods and means of production of works on new construction and reconstruction of existing buildings and structures of civil and industrial use;

Theoretical and experimental studies of the effectiveness of technological processes of construction and reorganization of construction objects;

Identification of modern patterns and trends through modeling and optimization of organizational and technological building solutions;

\footnotetext{
${ }^{*}$ Corresponding author: r.kazarian@mail.ru
} 
Development of new methods and improvement of existing methods of organizational and technological design of new construction and reconstruction of previously erected buildings and structures;

Increasing the managing efficiency of the organizational and technological design of the reorganization processes and its subsequent implementation.

The quality of the erection or construction reorganization of the facility mainly starts at the pre-project stage. Therefore, it is important at this stage to have a maximally concretized image of a new or reorganized object and ways of real achievement of the required quality, timing of implementation and the degree of conformity to the level of modern consumer properties.'

At the end of the last century, our country formulated the problem of developing an integrated model (a virtual construction object) that collectively reflects the necessary qualities of the future object. Such a model was proposed to be organized as a structure of indicators, criteria for their assessment, and the interrelationships of all components in a set of characteristics of a construction object. The actual development of virtual models of reorganized and newly constructed construction objects was started only at the beginning of the $21^{\text {st }}$ century by the construction industry. The model of the virtual construction object has to "accompany" the construction object throughout its life cycle, but it is the most relevant at the pre-project stage [4-8].

\section{Materials and Methods}

The purpose of the scientific research and virtual modeling of a construction object and construction industry that erects or reorganizes it is a comprehensive study of the object, process or phenomenon, their structure, connections and relations on the basis of newly discovered or scientifically known principles and methods of cognition. The result of such a study is the receipt and introduction of new methods of organizing labor activity and its elements into the practice of construction industry $[9,10]$.

The tasks of virtual modeling include:

- Analysis of existing domestic and foreign developments of virtual models for the erection or construction of facilities;

- Analysis of existing domestic and foreign methods of organizational and technological design for erection or constructional reorganization of buildings and structures;

Study of the characteristics of control systems for the erection and construction of buildings and structures;

- Modeling of the objective function (criterion) of management at the pre-project stage of erection and constructional reorganization of buildings and structures;

- Development of organizational structures and technologies of construction industry in relation to the selected object-representative of erected or reorganized buildings and structures;

- The formation of a methodology for the development of elements of technical, technological and organizational support for the management of the erection or constructional reorganization of buildings and structures, taking into account certain technogenic impacts.

The indirect or direct impact influencing a natural system, which is inherent in technology as an artificial system (which is the result of human thought activity, then realized in its productive activity) is called technogenic. This impact inevitably leads to changes in the parameters of functioning of human and his environment. Such changes are registered organoleptically (using sensory organs of a person, i.e. sight, hearing, touch, smell, etc.) or revealed in the process of instrumental diagnostics or computer monitoring. 
The feasibility and necessity of modeling technogenic impacts as an important element of managing the construction reorganization of objects in order to ensure the competitiveness of the proposed organizational and technological solutions are caused by a variety of reasons: - Constant increase in the scale, complexity and interrelationships in the systems, which leads to an increase in the complexity of the control objects themselves;

- Increased uncertainty in the knowledge of actual technogenic impacts due to intentional or accidental distortion of information;

- Dynamics of changes in political decisions or economic situation in the country, legal and law framework of activities;

- Changes in ownership patterns and increased responsibility of the owner for the results of operations;

- Moral and physical aging of the active part of production assets and housing;

- Changes in the status of a person in the system, when often he is viewed not as a determining component, but as an expendable resource.

These reasons increase the urgency of predicting technogenic impacts, planning methods for their accounting and management with the aim of achieving the required level of competitiveness of proposed organizational and technological solutions for the reorganization of facilities.

\section{Results}

Concepts and definitions related to the field of technogenic impacts modeling are not established; they are at the stage of formation. Therefore, there are different versions of the definitions of the same concept.

Simulation of hard-to-formalize and expertly estimated control factors for constructional reorganization of objects is performed for a fixed set of objects that are compared in pairs in terms of their preference (desirability, importance, etc.), and the results of the comparison are written as a matrix of paired comparisons $\mathbf{A}=\left|\mathbf{a}_{\mathbf{i j}}\right| \mathbf{m} \times \mathbf{n}$, reflecting the emerging binary 'preference / indifference' relation on the $\mathbf{X}$ set. Elements of the A matrix are imposed with additional calibration constraints (power calibration using inverse-symmetric matrices in the Bern-Brook-Burkov object ordering model, based on the hierarchy analysis method, etc.) clearly linking in pairs symmetric $\mathbf{a}_{\mathbf{i}}$ and $\mathbf{a}_{\mathbf{j}}$ elements.

The solution of the problem is a process of gradual setting of priorities, i.e. numerical estimates of the importance (weight) of the element along the whole set of assessments that are consistently attributed to the elements of the hierarchy in a particular situation. All elements of a certain $\mathbf{k}$ level of a simple hierarchy of criteria, factors, solutions, or alternatives affect any element of the (k-1) level and through them affect elements of higher levels of the hierarchy. The measure of this influence is shown by the priorities of the elements that are quantitatively estimated by the experts' assessments that pair and estimate the elements of one level in points, filling the square 'matrix of assessments'.

Assessments placed in $\mathbf{A}$ matrix are considered 'perfect', and the matrix itself is 'consistent' if for all values of $\mathbf{i}, \mathbf{j}$, and $\mathbf{k}$ the condition $\mathbf{a}_{\mathbf{i k}}=\mathbf{a}_{\mathbf{i j j}} \times \mathbf{a}_{\mathbf{j k}}$ is valid. For any 'triad' (three-point logics) of perfect assessments, the law of logical consequence (transitivity) applies: if $\mathbf{C}$ follows from $\mathbf{B}$, and $\mathbf{D}$ follows from $\mathbf{C}$, then $\mathbf{D}$ must follow from $\mathbf{B}$.

If $\mathbf{w}_{\mathbf{i j}}=\left(\mathbf{w}_{\mathbf{i}}: \mathbf{w}_{\mathbf{j}}\right)>\mathbf{O}$ for all $\mathbf{i}$ and $\mathbf{j}$, then all assessments are perfect and the matrix is consistent. Then $\mathbf{A w}=\mathbf{\Sigma} \mathbf{j}\left(\mathbf{a}_{\mathbf{i j}}-\mathbf{W} \mathbf{j}\right)=(\mathbf{n}-\mathbf{w i})=\mathbf{n w}$, where $\mathbf{w}=\mathbf{( w i )}$ is the $\mathbf{n}$-dimensional significance (weights) vector, $\lambda \max =\mathbf{n}$ is the Frobenius number, i.e. the senior modulo simple eigenvalue, and $\mathbf{w}$ is the unique eigenvector of the $\mathbf{A}$ matrix that corresponds to it (accurate to a constant factor).

For any matrix, its trace (the sum of the diagonal elements) is equal to the sum of the eigenvalues $\boldsymbol{\Sigma} \lambda \mathbf{i}=\mathbf{S p} \mathbf{A}=\boldsymbol{\Sigma} \mathbf{a}_{\mathbf{n}}$ (in the left sum, each $\lambda \mathbf{i}$ is taken taking considering its 
multiplicity). Therefore, for any inversely symmetric matrix $\boldsymbol{\Sigma} \lambda \mathbf{i}=\mathbf{n}$, and for the matched matrix, all e' eigenvalues, except for the Frobenius number, are zero (since e' rank is one).

Another problem is the sufficiency and completeness of the quality assessment of activity in functional systems for the erection and constructional reorganization of facilities. Kurt Godel's incompleteness theorems state that in theory, the attempt to 'describe' (formalize and evaluate) a system (as a set of elements that are in relationships and connections with each other) from within completely and consistently at the same time is doomed to failure.

There are true, but indefensible, judgments and controversial questions, the answers to which are required to be sought outside the system under investigation. In practice, the 'descriptions' of the structure, interrelations and technologies of the system's activities that regulate the level of the quality of its functioning are the result of an arrangement on the limitations organized by the methods of 'consensus' (agreement, unanimity of participants in the discussion when adopting the 'description' as a whole) or 'imposed consensus', similar to the administrative and command rationing.

In the infography, the procedure for organizing such a 'description' is simulated by a triad (three points) as a compromise between three interrelated concepts. Most often, the 'needopportunity-sufficiency (NOS)' and 'sufficiency-completeness-redundancy (SCR)' triads are used.

The system 'man-technology-environment (MTE)' models functional systems of construction industry, forms their internal 'descriptions' as a compromise of these triads, demonstrates a sufficiently high level of quality of functioning. Therefore, the question of the relationship between the concepts of 'sufficiency' and 'completeness' of the MTE system descriptions is relevant, as well as the construction of a basic infographic model on which these two concepts can be distinguished.

In order to unambiguously formalize and evaluate the local MTE system, it must be considered as a subsystem of a larger construction production system (metasystem). On an intrasystem level, the system can be: stable, steady (self-sufficient); not stable, not steady; on a non-systemic (suprasystem) level: completely formalized, partially formalized.

\section{Discussion}

A modern organizational and technological project for the construction of a building object, unlike the sum of a constructional and planning project of a building, projects of construction organization (PCO), projects of construction works (PCW), projects of work organization (PWO), and etc., which were separately developed during the pre-perestroika period, is formed as material and production scheduling system.

The functioning of this system is aimed at achieving a common goal that synthesizes the local goals of individual participants in the investment and innovative construction project, and is determined by the target function (criterion) of the management of the construction of the building object. The composition, content, and procedures of management design have changed; new organizational forms, methods, and means have been introduced to implement the tasks and functions of managing the construction or reorganization of the facility. First of all, this concerns the pre-project (initial) design stage.

The object of design is not a separate function or the engineering subsystem of the dwelling, but the development of organizational structures and technologies for building reconstruction with reference to the selected object-representative of the reconstructed buildings and structures. Such an object requires the development of management of the object creation components, which should cover the entire investment and innovative construction project taking into account its social, environmental, engineering and other subsystems. The subject of design is not a general contractor construction organization, but a special organizational structure for the management of the creation and operation of a 
building object (for example, a developer) that operates throughout the life cycle of the structure as a socio-technical project and includes diagnostic models of technogenic impacts $[3,5,7]$.

Diagnostic models are the models of technogenic influences, the research and use of which provide information on the causes of the identified problems. Using such diagnostic models, by analogy with dynamic infographic models of monitoring the parameters of the system and its components, dynamic series (sequences in time) of the predicted retrospective values of the parameters of technogenic impacts are constructed.

Visual displays of such dynamic series (infographic models) are known as 'trends'.

All known varieties of problem oriented forms of planning (for instance, strategic, business planning, perspective, current, calendar planning, etc.) are applicable to the area of technogenic impacts arising in the process of building reconstruction in a stationary environment. Business planning is distinguished from them by the fact that the decision is made with regard to the implementation of the system as a whole in one of the segments of the market of the reconstructed construction products. Scheduling is applicable to individual components of the system for a minimum period of time (work operation, work shift, etc.)

Forecasts of possible technogenic impacts and their results with regard to the functioning of the system as a whole or its individual components allow, with their evidentiary reliability, minimizing the time and money spent on the choice and justification of the most competitive organizational and technological solutions for building reconstruction of facilities (including a person as an element of the labor construction process and the priority component of the system).

Such forecasts are distinguished by:

- depth level (methods of functional, structural or parametric prediction, as well as complex methods);

- type of information (expert, functional and logical, structural, mathematical, complex forecasting or forecasting based on engineering diagnostics and intellectual monitoring of the system);

- the period of 'anticipation' (operational, short-term, medium-term, far-term, and long-term forecasts).

Each of these systematizations implies a separate approach to the choice of a method for predicting technogenic impacts in the system.

The most interesting is the searching functional and parametric operational and shortterm forecasting of the effect from the technogenic impacts in real time, carried out on the basis of the data of engineering diagnostics and intellectual monitoring in the process of mathematical modeling of the processes of the building reconstruction of objects, considered as a system.

This does not mean that the hierarchy of priorities for selecting modeling methods remains unchanged; it is continuously transformed by spontaneously changing reasons (economic, organizational and technological, voluntary, etc.).

When analyzing the possibility of technogenic impacts in the process of building reconstruction of the objects in terms of the degree of the conditions certainty, the following types of modeling methods are distinguished:

- with deterministic (certain) conditions;

- with random conditions having one of the known probability distributions;

- with uncertain conditions, in which a reasonable opponent counteracts (intentional technogenic impact).

Processes of organization and technology of interaction of system components that act as elements of the labor process of construction industry, which have been sufficiently studied, can be mathematically modeled and implemented, given the often encountered limitation: the possible intentional distortion of information. 
The complexity of solving the problem of predicting the effect of technogenic impacts in the process of building reconstruction of facilities on the competitiveness of the proposed variants of reorganization raises the need for a phased consideration of the factors contributing to the achievement of the necessary level of competitiveness, which include:

- Ensuring the reliability of the 'man-technology-environment, MTE' system;

- The use of a comprehensive innovative approach to the reorganization of facilities;

- Prioritization of system components;

- Development of information technology for engineering diagnostics of habitat, including fixation and mapping of technogenic impacts in the reconstructed buildings;

- Development of mathematical and infographic models of the impact of technogenic factors and changes in human functioning in the system.

The most significant influence is made by:

- Increasing the variety of modeling methods generated by the growing number and complexity of objects and practical tasks of building reconstruction (currently, there are more than one hundred methods, and acquaintance with them by searching takes time that the engineer may not have in the new business environment);

- Increasing 'mobility' (dynamics) of the market environment.

To ensure multi-criteria scheduling for the use of the organization resources of the reorganization of construction projects, it is necessary:

- To form a systematic approach to the scheduling of construction industry, taking into account the influence of external and internal factors of construction industry, the entire possible set of resources involved and based on the cybernetic management model;

- To systematize the types of used calendar 'plan-schedules, PS' (term by V.S. Gusakova) and methods of scheduling;

- To substantiate the basic principles of constructing dynamic models of construction industry, taking into account the simulation of the parameters of the construction system: the expedient use of a favorable period of the year and the minimization of real working time resources.

The peculiarity of the formation and use of PS scheduling and management is the need to take into account a certain dependence of the amount of work time resources on the calendar terms of construction and installation works (CIW).

If the deadlines for performing certain stages of the building reconstruction are not known, it is impossible to determine and initially set the main parameter of the scheduling the required resource of working time.

If the calendar plan preserves the structure of the normalized one, then the increase in resources for the execution of various types of construction and installation works is proportional to the duration of their implementation.

Such a pattern objectively leads the PS developer or specialist, who performs the function of monitoring the progress of the construction and installation works planned by PS, to the need to include the stage of adjustment of PS in the process after optimizing the use of the entire set of the involved resources of the construction reorganization.

In the process of such optimization, the PS developer or specialist, who performs the function of monitoring the progress of the planned PS of construction and installation works, binds the real parameters of the reorganization to the normalized values of normative documents.

The process of formation of object calendar PS is divided into several successively executed stages. They are:

- Collection, processing, and analysis of raw data in the form of regulatory parameters and constraints;

- Making decisions on the degree of detail and the results of assessing the reliability of the parameters; 
- Formation and calculation of variants of object scheduling on a normalized scale;

- Adjustment by transferring the object calendar PS from the normalized to the real time and the resources used scale;

- Multi-level optimization by the most significant criteria and resources by calculation and/or expert methods;

- Final formation of the project calendar PS taking into account all the above-mentioned stages and taking it as the basic optimal deterministic one.

The periods of deployment of subsequent CIW in real time and the resources used in this case are determined by linking the normalized values to the calendar time, taking into account their effect on the actual duration of the CIW.

As is known from the theory of the mass organization of construction works, the total duration of the implementation of the real object calendar plan of the CIW is defined as the sum of the real periods of the successively deployed CIW (starting from the second) and the time resource of the last type of CIW (all of them must be converted into real time and resources of the work fronts).

It is obvious that for the formation of such an object schedule for the CIW on the building reconstruction of an object, it is necessary to use the method of successive approximations realized with the help of computer programs (the number of such programs is sufficiently large; they are oriented not only to the continuous method of mass use of resources, but also to the method of continuous development of the work fronts and the critical path method).

Integrated PS' of the scheduling are formed from several object PS' with or without disturbance of their structure. Objects of new construction, reorganization, reconstruction, fixing, modernization, etc. can be included in one integrated PS', which leads to the need to use different forms of PS' and the means of their implementation.

The most important requirement of a complex calendar PS is the inadmissibility of work stoppage (freezing of any of the construction objects considered in it); this is due not only to the inevitably arising violations of technology and the organization of construction production, but also to economic losses [8-10].

These violations and losses are leveled out in different ways, identifying and implementing:

- Different dependencies between the timeframes for the production of CIW and the required resources of working time, as well as between the duration and cost of restrictions;

- Making decisions on the degree of detail and the results of assessing the reliability of the parameters;

- Forming and calculating variants of object scheduling on a normalized scale;

- Making adjustments by transferring the object calendar PS from the normalized to the real time scale and the resources used;

- Performing multi-level optimization of the most significant criteria and resources by calculation and/or expert methods;

- Finally forming the project calendar PS taking into account all the above-mentioned stages and adopting it as the basic optimal deterministic planning document.

The periods of deployment of subsequent construction and installation works (CIW) in real time and used resources scale are determined by linking the normalized values to the calendar time, taking into account their effect on the actual duration of the construction work.

Dependences between the calendar terms of CIW production and the necessary resources of working time, between the duration and the cost of CIW allow us to formulate two optimization tasks that require defining the scheduling parameters. They are:

- Production of construction and installation works in which the cost of work is minimal;

- The construction operations in which the minimum resources for the implementation of construction and installation work will be achieved. 


\section{Conclusions}

For scheduling, the utility of various rearrangements consists in the formation of flows with minimal reserves (breaks); these reserves can also be used to reduce the number of contractors. Algorithm for the formation of PS, based on the solution of inconsistent time and resource constraints by the method of uncertain resource coefficients, allows optimizing the number of CIW performers and allows reducing the risk of untimely performance of CIW.

As a result of the conducted studies, the exponential distribution of random work durations is justified, which not only reflects the untimeliness of the CIW implementation, but also makes it possible to obtain an array of random durations based on a single statistical parameter, which is the average untimely performance of the work.

\section{References}

1. A. A. Volkov, V. O. Chulkov, G. O. Chulkov, R. R. Kazaryan, O. N. Kyzina, Advanced Materials Research, 1065-1069, 2401-2404 (2015) doi: 10.4028/www.scientific.net/AMR.1065-1069.2401.

2. A. A. Volkov, V. O. Chulkov, G. O. Chulkov, R. R. Kazaryan, O. N. Kyzina, Advanced Materials Research, 1065-1069, 2405-2408 (2015) doi: 10.4028/www.scientific.net/AMR.1065-1069.2405.

3. A. A. Volkov, V. O. Chulkov, G. O. Chulkov, R. R. Kazaryan, O. N. Kyzina, Advanced Materials Research, 1065-1069, 2405-2408 (2015) doi: 10.4028/www.scientific.net/AMR.1065-1069.2409.

4. R. R. Kazaryan, MATEC Web of Conferences, 193, 01009 (2018)

5. R. R. Kazaryan, MATEC Web of Conferences, 193, 04023 (2018)

6. A. Slavin, S. Sinenko, N. Yoshin, IOP Conference Series: Materials Science and Engineering, Construction - The Formation of Living Environment, 21, 62040 (2018)

7. S. Sinenko, A. Ahmetgaliev, A. Slavin, IOP Conference Series: Materials Science and Engineering, Construction - The Formation of Living Environment, 21, 062039 (2018)

8. B. Zhadanovsky, S. Sinenko, E3S Web of Conferences "High-Rise Construction 2017, HRC 2017", 33, 03077 (2018)

9. S. Novikov, B. Zhadanovsky, S. Sinenko, MATEC Web of Conferences, 22. 06011 (2018)

10. S. Novikov, B. Zhadanovsky, S. Sinenko, MATEC Web of Conferences, 22. 09007 (2018) 